\title{
INTERNATIONALER HANDEL MIT WAREN
}

Seit ihrer Gründung ist die OECD bestrebt, den internationalen Handel zu fördern, denn sie sieht darin einen wirksamen Mechanismus zur Stärkung des Wirtschaftswachstums und zur Hebung des Lebensstandards. Ein intensiverer Handel kommt den OECD-Mitgliedsländern ebenso wie deren Handelspartnern in der übrigen Welt zugute.

\section{Definition}

Nach den Empfehlungen der Vereinten Nationen erfassen die internationalen Warenhandelsstatistiken sämtliche Waren, die den Bestand eines Landes an materiellen Ressourcen vergrößern bzw. verringern, indem sie in sein Wirtschaftsgebiet verbracht werden (als Einfuhren) bzw. dieses verlassen (als Ausfuhren). Waren, die durch ein Land transportiert oder die vorübergehend ein- bzw. ausgeführt werden (mit Ausnahme von Waren zur Be- oder Verarbeitung im In- oder Ausland), sind in den Statistiken des Warenhandels nicht berücksichtigt.

\section{Vergleichbarkeit}

Alle OECD-Länder wenden die Empfehlungen der Vereinten Nationen an, soweit es die vorliegenden Datenquellen zulassen. Es bestehen einige - meist kleinere - Unterschiede zwischen den Ländern bei der Erfassung bestimmter Kategorien von Transaktionen, wie z.B. Versandhandel, Import und Export von Militärausrüstungen im Rahmen von Verteidigungsabkommen, Handel mit Meeresprodukten von Schiffen unter

\section{Überblick}

Für alle in der Tabelle aufgeführten Länder ist der Warenhandel im Betrachtungszeitraum stetig gewachsen. Zwischen 2008 und 2009 machten sich jedoch die Auswirkungen der globalen Finanzkrise auf den Warenhandel bemerkbar. Die Auswirkungen der Krise auf die Einfuhren fielen für China, die Schweiz, Indien und Australien in relativer Rechnung moderater aus, da die Einfuhren dort um weniger als $15 \%$ gesunken sind. Für die Russische Föderation und Island waren die Auswirkungen insofern gravierender, als die Einfuhren dieser Länder um mehr als $35 \%$ geschrumpft sind. Die Ausfuhren wurden zwischen 2008 und 2009 ebenfalls durch die Krise beeinträchtigt, sie sind z.B. in Finnland und der Russischen Föderation um über 35\% eingebrochen, in Indien, Irland, Korea, der Schweiz und Indonesien sind sie aber um weniger als 15\% zurückgegangen.

Das Defizit in der Warenhandelsbilanz ist während des in der Tabelle erfassten Zeitraums in mehreren OECD-Ländern gestiegen. Das war z.B. der Fall in den Vereinigten Staaten, Frankreich, dem Vereinigten Königreich und der Türkei. China und die Russische Föderation konnten hingegen weiterhin einen Überschuss in der Warenhandelsbilanz ausweisen.

Erwähnenswert ist die starke Verschlechterung der Warenhandelsbilanz Japans, die 2011 zum ersten Mal in dreißig Jahren ins Defizit gerutscht ist, was aller Wahrscheinlichkeit nach durch die Nachwirkungen des Erdbebens und des Tsunamis bedingt war. inländischer Flagge auf Hochseegewässern sowie die Ein- und Ausfuhr von Waren in bzw. aus Zollausschlussgebieten.

Der Ausfuhrwert wird gewöhnlich FOB (free on board) berechnet, außer von den Vereinigten Staaten, die ihn FAS (free alongside ship) angeben, d.h. ohne die Verladekosten. Die Einfuhrwerte werden von den meisten Ländern CIF (cost, insurance and freight) berechnet, d.h. zusätzlich zum Rechnungswert werden Versicherungs- und Frachtkosten bis zur Grenze des Einfuhrlandes einbezogen. Kanada deklariert seine Einfuhren jedoch zu FOB-Werten.

Infolge der Schaffung des europäischen Binnenmarkts im Jahr 1993 haben die Daten für den Intra-EU-Handel etwas an Präzision eingebüßt, da seitdem keine Zollunterlagen mehr verfügbar sind, in denen sämtliche Ein- und Ausfuhren aufgezeichnet wären. Es ist zu beachten, dass die Erfassung der OECD-Daten größtenteils nach den Empfehlungen der Vereinten Nationen erfolgt, während die Außenhandelsstatistiken von Eurostat gemäß dem Gemeinschaftskonzept erstellt werden und mit den hier dargestellten nicht uneingeschränkt vergleichbar sind.

„OECD insgesamt“ umfasst erst ab 1999 alle 34 Mitgliedsvolkswirtschaften.

\section{Quelle}

- OECD (2012), International Trade by Commodity Statistics, OECD Publishing.

- Vereinte Nationen (2012), United Nations Commodity Trade Statistics (Datenbank).

\section{Weitere Informationen}

\section{Analysen}

- OECD (2011), Globalisation, Comparative Advantage and the Changing Dynamics of Trade, OECD Publishing.

- OECD (2006), Aid for Trade: Making it Effective, The Development Dimension, OECD Publishing.

- OECD (2006), Trade Based Money Laundering, OECD Publishing.

- OECD (2005), Trade and Structural Adjustment: Embracing Globalisation, OECD Publishing.

\section{Statistiken}

- OECD (2012), Monthly Statistics of International Trade, OECD Publishing.

\section{Zur Methodik}

- OECD (2012), International Trade by Commodity Statistics, OECD Publishing.

- Vereinte Nationen (2004), International Merchandise Trade Statistics: Compilers Manual, Vereinte Nationen.

\section{Online-Datenbanken}

- International Trade by Commodity Statistics.

- Monthly Statistics of International Trade.

Websites

- OECD International Trade and Balance of Payments Statistics, www.oecd.org/std/its. 


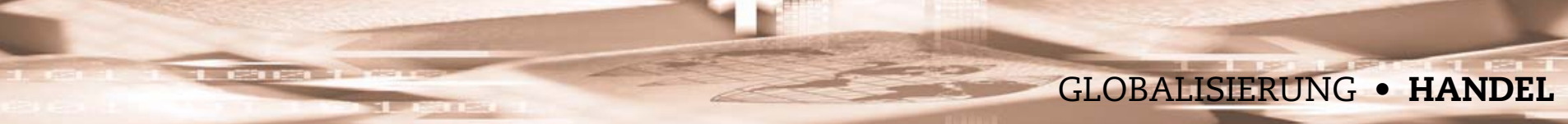

INTERNATIONALER HANDEL MIT WAREN

\section{Internationaler Handel mit Waren}

Mrd. US-Dollar

\begin{tabular}{|c|c|c|c|c|c|c|c|c|c|c|c|c|}
\hline & \multicolumn{4}{|c|}{ Handelssaldo } & \multicolumn{4}{|c|}{ Einfuhren } & \multicolumn{4}{|c|}{ Ausfuhren } \\
\hline & 2000 & 2005 & 2010 & 2011 & 2000 & 2005 & 2010 & 2011 & 2000 & 2005 & 2010 & 2011 \\
\hline Australien & -4.0 & -12.8 & 18.6 & 11.3 & 67.8 & 118.9 & 193.3 & 234.3 & 63.8 & 106.0 & 211.8 & 245.6 \\
\hline Belgien & 13.5 & 13.8 & 21.0 & 12.7 & 171.7 & 320.2 & 390.1 & 465.2 & 185.2 & 334.0 & 411.1 & 477.9 \\
\hline Chile & 1.6 & 10.4 & 13.1 & 6.5 & 16.6 & 29.5 & 56.2 & 74.9 & 18.2 & 39.9 & 69.4 & 81.4 \\
\hline Dänemark & 5.2 & 8.3 & 12.3 & 13.0 & 44.4 & 75.0 & 84.5 & 88.5 & 49.6 & 83.3 & 96.8 & 101.5 \\
\hline Deutschland & 54.8 & 197.3 & 204.3 & 221.9 & 495.4 & 779.8 & 1066.8 & 1260.3 & 550.2 & 977.1 & 1271.1 & 1482.2 \\
\hline Estland & -1.2 & -2.8 & -0.4 & -0.6 & 5.1 & 11.0 & 13.2 & 18.8 & 3.8 & 8.2 & 12.8 & 18.2 \\
\hline Finnland & 11.7 & 6.8 & 1.4 & -5.1 & 34.1 & 58.5 & 68.8 & 83.9 & 45.8 & 65.2 & 70.1 & 78.8 \\
\hline Frankreich & -8.5 & -41.6 & -87.5 & -119.3 & 304.0 & 476.0 & 599.2 & 700.9 & 295.6 & 434.4 & 511.7 & 581.5 \\
\hline Griechenland & -18.8 & -37.4 & -41.8 & -29.1 & 29.8 & 54.9 & 63.3 & 60.8 & 11.0 & 17.5 & 21.6 & 31.7 \\
\hline Irland & 25.6 & 39.7 & 57.8 & 62.3 & 50.7 & 70.3 & 60.5 & 67.1 & 76.3 & 110.0 & 118.3 & 129.3 \\
\hline Island & -0.7 & -1.9 & 0.7 & 0.5 & 2.6 & 5.0 & 3.9 & 4.8 & 1.9 & 3.1 & 4.6 & 5.3 \\
\hline Israel & -4.3 & -2.3 & -0.8 & -5.7 & 35.7 & 45.0 & 59.2 & 73.5 & 31.4 & 42.8 & 58.4 & 67.8 \\
\hline Italien & 1.9 & -11.9 & -39.9 & -34.3 & 238.1 & 384.8 & 486.6 & 557.5 & 239.9 & 373.0 & 446.8 & 523.2 \\
\hline Japan & 99.6 & 79.1 & 75.7 & -31.3 & 379.7 & 515.9 & 694.1 & 854.6 & 479.2 & 594.9 & 769.8 & 823.3 \\
\hline Kanada & 37.6 & 46.1 & -5.5 & -0.2 & 240.0 & 314.4 & 392.1 & 450.4 & 277.6 & 360.6 & 386.6 & 450.1 \\
\hline Korea & 11.8 & 23.2 & 41.2 & 30.8 & 160.5 & 261.2 & 425.2 & 524.4 & 172.3 & 284.4 & 466.4 & 555.2 \\
\hline Luxemburg & -2.8 & -4.9 & -6.5 & -8.9 & 10.6 & 17.6 & 20.4 & 25.3 & 7.9 & 12.7 & 13.9 & 16.3 \\
\hline Mexiko & -5.8 & -7.6 & -3.2 & -1.3 & 171.1 & 221.8 & 301.5 & 350.8 & 165.3 & 214.2 & 298.3 & 349.6 \\
\hline Neuseeland & -0.6 & -4.5 & 0.8 & 1.5 & 13.9 & 26.2 & 30.2 & 36.1 & 13.3 & 21.7 & 30.9 & 37.6 \\
\hline Niederlande & 5.4 & 36.9 & 52.7 & .. & 174.7 & 283.2 & 440.0 & .. & 180.1 & 320.1 & 492.6 & 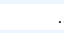 \\
\hline Norwegen & 25.5 & 48.3 & 54.1 & 68.5 & 34.4 & 55.5 & 77.3 & 90.8 & 59.9 & 103.8 & 131.4 & 159.4 \\
\hline Österreich & -5.2 & -2.2 & -5.7 & .. & 67.4 & 120.0 & 150.6 & .. & 62.3 & 117.7 & 144.9 & \\
\hline Polen & -17.2 & -12.2 & -17.1 & -19.7 & 48.8 & 101.5 & 174.1 & 203.0 & 31.6 & 89.4 & 157.1 & 183.3 \\
\hline Portugal & -15.6 & -23.1 & -26.5 & -21.4 & 39.9 & 61.2 & 75.2 & 80.3 & 24.4 & 38.1 & 48.8 & 58.9 \\
\hline Schweden & 14.2 & 18.9 & 9.7 & 11.2 & 73.1 & 111.4 & 148.4 & 176.0 & 87.4 & 130.3 & 158.1 & 187.2 \\
\hline Schweiz & -2.0 & 4.4 & 19.3 & 27.2 & 82.5 & 126.6 & 176.3 & 207.3 & 80.5 & 130.9 & 195.6 & 234.4 \\
\hline Slowak. Rep. & -0.9 & -2.4 & -0.4 & 1.8 & 12.7 & 34.2 & 64.4 & 76.7 & 11.8 & 31.9 & 64.0 & 78.5 \\
\hline Slowenien & -1.4 & -1.7 & -2.2 & -2.3 & 10.1 & 19.6 & 26.4 & 30.8 & 8.7 & 17.9 & 24.2 & 28.5 \\
\hline Spanien & -39.5 & -96.8 & -70.6 & .. & 152.9 & 289.6 & 318.2 & & 113.3 & 192.8 & 247.6 & . \\
\hline Tschech. Rep. & -3.2 & 1.7 & 6.5 & 11.6 & 32.2 & 76.5 & 125.7 & 150.5 & 29.1 & 78.2 & 132.1 & 162.1 \\
\hline Türkei & -26.7 & -43.3 & -71.6 & -105.9 & 54.5 & 116.8 & 185.5 & 240.8 & 27.8 & 73.5 & 114.0 & 134.9 \\
\hline Ungarn & -4.0 & -3.6 & 7.3 & 9.5 & 32.1 & 65.9 & 87.4 & 101.5 & 28.1 & 62.3 & 94.7 & 111.1 \\
\hline Ver. Königreich & -56.6 & -131.4 & -156.6 & -162.3 & 339.4 & 515.8 & 562.4 & 634.4 & 282.9 & 384.4 & 405.8 & 472.1 \\
\hline Ver. Staaten & -477.7 & -828.0 & -689.4 & -782.9 & 1258.1 & 1732.3 & 1966.5 & 2262.6 & 780.3 & 904.3 & 1277.1 & 1479.7 \\
\hline EU27 & & -157.8 & -204.7 & .. & .. & 1465.1 & 1990.5 & .. & .. & 1307.3 & 1785.8 & . \\
\hline OECD & -398.0 & -737.1 & -629.1 & .. & 4861.6 & 7493.9 & 9587.4 & .. & 4463.6 & 6756.8 & 8958.3 & \\
\hline Brasilien & -0.7 & 44.9 & 16.9 & 29.8 & 55.9 & 73.6 & 180.5 & 226.2 & 55.1 & 118.5 & 197.4 & 256.0 \\
\hline China & 24.1 & 102.0 & 181.8 & 155.0 & 225.1 & 660.0 & 1396.0 & 1743.4 & 249.2 & 762.0 & 1577.8 & 1898.4 \\
\hline Indien & -10.6 & -40.5 & -129.6 & .. & 52.9 & 140.9 & 350.0 & .. & 42.4 & 100.4 & 220.4 & \\
\hline Indonesien & 28.6 & 28.0 & 22.1 & 26.1 & 33.5 & 57.7 & 135.7 & 177.4 & 62.1 & 85.7 & 157.8 & 203.5 \\
\hline Russ. Föderation & 69.2 & 142.7 & 151.4 & 193.3 & 33.9 & 98.7 & 248.7 & 284.7 & 103.1 & 241.5 & 400.1 & 478.0 \\
\hline Südafrika & -0.5 & -8.0 & -8.7 & -6.8 & 26.8 & 55.0 & 80.1 & 99.7 & 26.3 & 47.0 & 71.5 & 93.0 \\
\hline
\end{tabular}

StatLink Aiाsस $h t t p: / / d x$. doi.org/10.1787/888932707268

Entwicklung der Warenhandelsbilanz

Jährliche Veränderung in Prozent

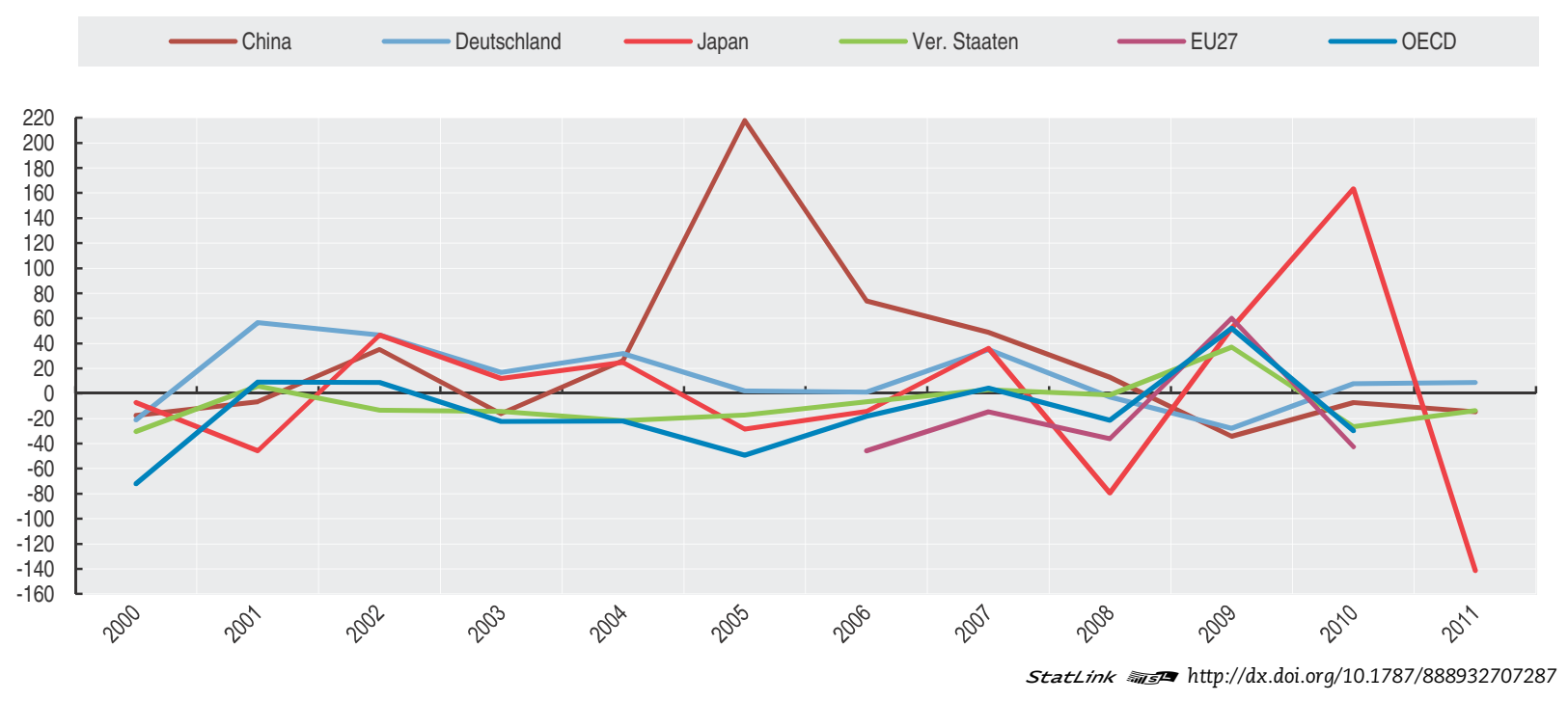




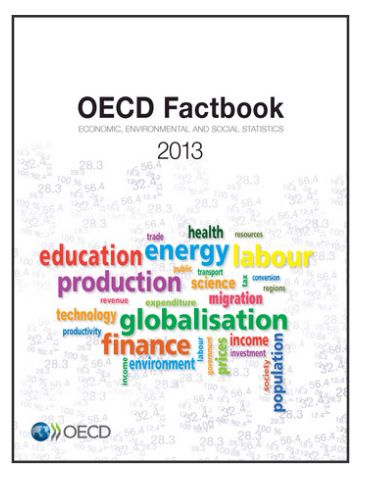

\section{From: \\ OECD Factbook 2013 \\ Economic, Environmental and Social Statistics}

Access the complete publication at:

https://doi.org/10.1787/factbook-2013-en

Please cite this chapter as:

OECD (2013), "Internationaler Handel mit Waren", in OECD Factbook 2013: Economic, Environmental and Social Statistics, OECD Publishing, Paris.

DOI: https://doi.org/10.1787/factbook-2013-31-de

Das vorliegende Dokument wird unter der Verantwortung des Generalsekretärs der OECD veröffentlicht. Die darin zum Ausdruck gebrachten Meinungen und Argumente spiegeln nicht zwangsläufig die offizielle Einstellung der OECD-

Mitgliedstaaten wider.

This document and any map included herein are without prejudice to the status of or sovereignty over any territory, to the delimitation of international frontiers and boundaries and to the name of any territory, city or area.

You can copy, download or print OECD content for your own use, and you can include excerpts from OECD publications, databases and multimedia products in your own documents, presentations, blogs, websites and teaching materials, provided that suitable acknowledgment of OECD as source and copyright owner is given. All requests for public or commercial use and translation rights should be submitted to rights@oecd.org. Requests for permission to photocopy portions of this material for public or commercial use shall be addressed directly to the Copyright Clearance Center (CCC) at info@copyright.com or the Centre français d'exploitation du droit de copie (CFC) at contact@cfcopies.com. 